\title{
INTERNATIONAL JOURNAL OF PRECLINICAL AND CLINICAL RESEARCH
}

RESEARCH ARTICLE

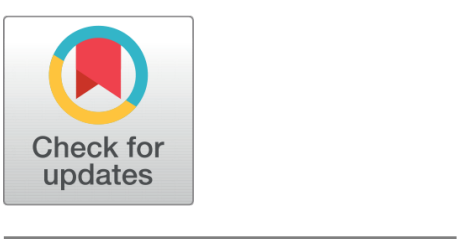

OPEN ACCESS

Received: 10.02.2021

Accepted: 01.04.2021

Published: 05.04.2021

Citation: Aswathi TV, Vickram , Divya D. (2021). Serum uric acid measurement and its correlation with urinary albumin-creatinine ratio in type-2 Diabetic subjects. International Journal of Preclinical \& Clinical Research. 2(1): 5-7. https://d oi.org/10.51131/IJPCCR/v2i1.5

Corresponding author.

drvickramkaali@gmail.com

Funding: None

Competing Interests: None

Copyright: ( 2021 Aswathi et al. This is an open access article distributed under the terms of the Creative Commons Attribution License, which permits unrestricted use, distribution, and reproduction in any medium, provided the original author and source are credited.

Published By Basaveshwara Medical College \& Hospital, Chitradurga, Karnataka

\section{ISSN}

Print: $X X X X-X X X X$

Electronic: $X X X X-X X X X$

\section{Serum uric acid measurement and its correlation with urinary albumin-creatinine ratio in type-2 Diabetic subjects}

\author{
T V Aswathi ${ }^{1}$, Vickram ${ }^{2 *}$, D Divya ${ }^{3}$ \\ 1 Post graduate, Kannur University, Palayad, Kerala, India \\ 2 Professor, Department of Biochemistry, Basaveshwara Medical College and Hospital, \\ Chitradurga, 577502, Karnataka, India \\ 3 Associate Professor, Department of Biochemistry, Basaveshwara Medical College and \\ Hospital, Chitradurga, 577502, Karnataka, India
}

\section{Abstract}

Increased serum uric acid (SUA) is known to play a critical role in the development of type-2 diabetes mellitus (T2DM) as well as in progression of its complications. Hyperuricemia is an independent risk factor for kidney dysfunction in patients with diabetes. The appearance of albuminuria is the first sign of kidney damage and the mechanisms of hyperuricemia-induced renal injury mainly include oxidative stress, endothelial dysfunction, renal fibrosis, and inflammation. To determine the possible association of SUA with urinary albumin-creatinine ratio (ACR) in type-2 diabetic subjects, data with lab investigations of SUA and urinary ACR of 95 confirmed and known cases of T2DM were collected from the laboratory medicine department, DMWIMS Hospital, Wayanad Kerala. Based on the urinary albumin excretion values, diabetic subjects were divided into three groups (Normoalbuminuric, microalbuminuric \& macroalbuminuric groups). A significant increase in mean ACR from group $-1 \rightarrow$ group $-2 \rightarrow$ group- 3 was observed which is proportional to increase in mean SUA levels suggesting that increase in SUA may be responsible for increased urinary albumin excretion. This study shows a positive correlation between SUA and ACR.

Keywords: Serum uric acid; diabetes mellitus; hyperuricemia; kidney damage; T2DM; albuminuria; urinary albumin-creatinine ratio; diabetic nephropathy

\section{Introduction}

Uric acid (UA) is produced in the liver as an end product of purine catabolism and approximately $1 / 3$ of it is degraded in the gut and $2 / 3$ is renally excreted. ${ }^{(1,2)}$ Increased serum uric acid (SUA) or hyperuricemia is clinically defined as SUA level $\geq 7 \mathrm{mg} / \mathrm{dL}$ in men and postmenopausal women and $\geq 6 \mathrm{mg} / \mathrm{dL}$ in premenopausal women. ${ }^{3}$ Hyperuricemia has been linked with many clinical conditions. ${ }^{(4)}$ It is known to play a 
critical role in the development of type-2 diabetes mellitus (T2DM) as well as in progression of its complications. ${ }^{(5-7)}$ Hyperuricemia is an independent risk factor for kidney dysfunction in patients with DM. ${ }^{(8)}$ The mechanisms of hyperuricemia-induced renal injury mainly include oxidative stress, endothelial dysfunction, renal fibrosis, and inflammation. ${ }^{(3)}$ Recent studies have reported that hyperuricemia is associated with kidney damage independent of hypertension in diabetic patients. ${ }^{(9)}$ On the other hand, higher levels of serum insulin may decrease uric acid clearance by the kidneys. ${ }^{(9)}$ As a rule, hyperinsulinemia is the basis of T2DM pathophysiology. ${ }^{(10)}$. Therefore, diabetic patients are more prone to uric acid injury. It is also reported that hyperuricemia may positively influence urinary albumin excretion by decreasing glomerular filtration rate (GFR) and increases the risk of kidney failure. ${ }^{(11,12)}$ We know that albuminuria is an early marker for renal damage, and hence its measurement is important for the detection of diabetic kidney disease (DKD) or diabetic nephropathy (DN). This study was undertaken to determine the possible association of SUA with urinary albumin-creatinine ratio (ACR) in type-2 diabetic subjects.

\section{Materials and Methods}

\section{Study Setting \& Data Source}

A retrospective observational study was conducted in DM WIMS Hospital, Wayanad, in collaboration with Department of Medical Laboratory Technology, School of Health Sciences, Palayad, Kannur University, Kerala, during the period from July 2019 to August 2019. Approval has been taken from Institutional Research Committee [Ref.No: CAU/11/SHS/RC(1)/19, dated 10/06/19] for conducting this study. One year data (from Jan 2018-Jan 2019) of confirmed and known cases of T2DM were collected from the hospital laboratory medicine department for the present study.

\section{Study Subjects}

A total of 95 type 2 diabetic subjects (43 Males +52 Females) with lab investigations of SUA and urinary ACR done, were randomly selected based on inclusion and exclusion criteria.

Inclusion criteria: Patients $>40$ years of age with known case of T2DM were included in this study.

Exclusion criteria: Diabetic patients with end stage renal disease, patients who were on dialysis, patients who were on treatment with uric acid lowering agents, diuretics, or alcoholic beverages, history of gout, acute febrile illness, urinary tract infection or cancer were excluded from the study.

\section{Study Parameters}

SUA was measured by enzymatic colorimetric assay. Albuminuria is measured by estimating urinary ACR [by measuring urinary albumin by BCG dye-binding method and urinary creatinine by enzymatic method and calculating the albumin/creatinine ratio] which is a preferred method for spot urine/early morning urine samples. All estimations were performed using Roche Cobas Integra 400 Plus fully automated analyser.

\section{Study Groups}

Based on the urinary albumin excretions values, expressed as urinary albumin-creatinine ratio (mg albumin/g creatinine), the Type-2 diabetic subjects were divided into three groups.

1. Normoalbuminuric group (Group-1) $=$ ACR $<30 \mathrm{mg} / \mathrm{g}$

2. Microalbuminuric group (Group-2) $=$ ACR 30-300 $\mathrm{mg} / \mathrm{g}$

3. Macroalbuminuric group (Group-3) $=\mathrm{ACR}>300 \mathrm{mg} / \mathrm{g}$

\section{Statistical Evaluation}

Statistical data analysis was done using SPSS software version 24 (IBM, Armonk, NY, USA). The values are expressed as mean \pm SD and Spearman's rho method was employed to assess the correlation of SUA with ACR. P value $-\mathrm{P}<0.05$ was considered statistically significant.

\section{Results}

Results of the present study is summarized in Table 1.

Table 1. Table showing Age, Sex, levels of SUA and ACR,in group-1, group-2, and group-3 diabetic subjects.

\begin{tabular}{llll}
\hline Parameters & Group-1 & Group-2 & Group-3 \\
\hline Age & $58.76 \pm 13.01$ & $57.15 \pm 13.82$ & $62.32 \pm 13.39$ \\
Male & 18 & 14 & 11 \\
Female & 19 & 19 & 14 \\
Total & 37 & 33 & 25 \\
SUA & $4.56 \pm 1.33$ & $7.21 \pm 1.14$ & $8.18 \pm 1.48$ \\
ACR & $15.56 \pm 6.89$ & $119.78 \pm 70.71$ & $658.77 \pm$ \\
& & & 271.97 \\
\hline
\end{tabular}

Note: values are expressed as mean $\pm \mathrm{SD}$.

\section{Discussion}

Both animal and human studies have suggested that hyperuricemia is strongly associated with renal damage in DM. ${ }^{(6,13)}$ The various mechanisms by which hyperuricemia initiates renal damage in diabetics are well recognized. ${ }^{(3,6)}$ One such 
mechanism is activation of renin angiotensin-aldosterone system (RAAS). In diabetes RAAS activation causes a range of pathological changes including vascular dysfunction, high intraglomerular pressure, inflammation, and so on, leading to cardiovascular and renal complications. ${ }^{(14)}$ From table1 it is clear that there is significant increase in mean ACR from group- $1 \rightarrow$ group- $\rightarrow$ group- 3 which is proportional to increase in SUA levels suggesting that increase in SUA is responsible for increased urinary albumin excretion. The increase in albumin excretion is may be due to decrease in GFR which in turn is due to increased SUA. ${ }^{(11)}$ In the present study, a significant $(\mathrm{p}<0.01)$ positive correlation was found between SUA and ACR. Our study is consistent with the studies of Fukui, et al. ${ }^{(8)}$ where they have reported a positive correlation of serum uric acid and urinary albumin excretion in 343 men with T2DM. Another study has shown that SUA and microalbuminuria were significantly and positively correlated with nephropathy in type-2 diabetic patients. ${ }^{(15)}$ Siu and colleagues ${ }^{(16)}$ reported lowering serum uric acid level in patients with hyperuricemia was associated with regression of kidney disease. Fu et al. in a study on Chinese diabetic patients found that hyperuricemia was significantly associated with abnormal albuminuria in patients without diuretics or use of uricosuric agents or alcohol. ${ }^{(17)}$ Since appearance of albuminuria is the first sign of kidney damage and onset of DN in patients with DM, measuring SUA in patients with T2DM and correcting the levels if there is hyperuricemia may prove to be beneficial for preventing the progression of T2DM to $\mathrm{DN}$.

\section{Limitations of the Study}

Although this study shows a positive correlation of SUA with urinary ACR in diabetic patients, it fails to account for authenticity of the same and generalizability; due to its retrospective design, poor sample size, limited analysis of variables, and not accounting for confounding variables.

\section{Conclusion}

This study shows a positive correlation between SUA and urinary ACR ratio suggesting the importance of SUA levels as a predictor of albuminuria in type- 2 diabetic subjects. The pathogenic role of hyperuricemia in renal injury, in increasing urinary albumin excretion rate and in progression of diabetes to its chronic complication, nephropathy, is worthy of further investigation. Also, we call for further research studies on use of SUA lowering drugs in various clinical conditions witnessing hyperuricemia.

\section{References}

1) Bonakdaran S, Hami M, Shakeri MT. Hyperuricemia and albuminuria in patients with type 2 diabetes mellitus. Iran J Kidney Dis. 2011;5(1):2125.

2) Bobulescu IA, Moe OW. Renal Transport of Uric Acid: Evolving Concepts and Uncertainties. Advances in Chronic Kidney Disease. 2012;19:358-371. doi:10.1053/j.ackd.2012.07.009.

3) Su HY, Yang C, Liang D, Liu HF. Research Advances in the Mechanisms of Hyperuricemia-Induced Renal Injury. Biomed Res Int. 2020;2020:5817348.

4) Jin M, Yang F, Yang I, Yin Y, Luo JJ, Wang H, et al. Uric acid, hyperuricemia and vascular diseases. Front Biosci. 2012;17:656-69.

5) Bhole V, Choi JWJ, Kim SW, de Vera M, Choi H. Serum Uric Acid Levels and the Risk of Type 2 Diabetes: A Prospective Study. The American Journal of Medicine. 2010;123(10):957-961. doi:10.1016/j.amjmed.2010.03.027.

6) Xiong Q, Liu J, Xu Y. Effects of Uric Acid on Diabetes Mellitus and Its Chronic Complications. Int J Endocrinol. 2019;p. 9691345.

7) Kodama S, Saito K, Yachi Y, Asumi M, Sugawara A, Totsuka K, et al. Association Between Serum Uric Acid and Development of Type 2 Diabetes. Diabetes Care. 2009;32:1737-1742. doi:10.2337/dc09-0288.

8) Fukui M, Tanaka M, Shiraishi E, and Hosoda H HI, M A, et al. Serum uric acid is associated with microalbuminuria and subclinical atherosclerosis in men with type 2 diabetes mellitus. Serum uric acid is associated with microalbuminuria and subclinical atherosclerosis in men with type 2 diabetes mellitus Metab. 2008;57(5):625-634. doi:10.1016/j.metabol.2007.12.005.

9) Tseng $\mathrm{CH}$. Correlation of uric acid and urinary albumin excretion rate in patients with type 2 diabetes mellitus in Taiwan. Kidney Int. 2005;68:796-801.

10) Bo S, Cavallo-Perin P, Gentile L, Repetti E, Pagano G. Hypouricemia and hyperuricemia in type 2 diabetes: two different phenotypes. European Journal of Clinical Investigation. 2001;31(4):318-321. doi:10.1046/j.1365-2362.2001.00812.x.

11) Cosmo SD, Viazzi F, Pacilli A, Giorda C, Ceriello A, Gentile $S$, et al. AMD-Annals Study Group. Serum Uric Acid and Risk of CKD in Type 2 Diabetes. Clin J Am Soc Nephrol. 2015;10(11):1921-1930.

12) Tsai CW, Lin SY, Kuo CC, Huang CC. Serum Uric Acid and Progression of Kidney Disease: A Longitudinal Analysis and Mini-Review. PLoS One. 2017;12(1):e170393.

13) Behradmanesh $S$, Horestani MK, Baradaran A, Nasri H. Association of serum uric acid with proteinuria in type 2 diabetic patients. J Res Med Sci. 2013;18(1):44-50.

14) Zatz R, Meyer TW, Rennke HG, Brenner BM. Predominance of hemodynamic rather than metabolic factors in the pathogenesis of diabetic glomerulopathy. Proceedings of the National Academy of Sciences. 1985;82(17):5963-5967. doi:10.1073/pnas.82.17.5963.

15) Latif H, Iqbal A, Rathore R, Butt NF. Correlation between Serum Uric Acid Level and Microalbuminuria in Type-2 Diabetic Nephropathy. Pakistan Journal of Medical Sciences. 2017;33(6):1371-1375. doi:10.12669/pjms.336.13224.

16) Siu YP, Leung KT, Tong MKH, Kwan TH. Use of Allopurinol in Slowing the Progression of Renal Disease Through Its Ability to Lower Serum Uric Acid Level. American Journal of Kidney Diseases. 2006;47(1):5159. doi:10.1053/j.ajkd.2005.10.006.

17) $\mathrm{Fu} \mathrm{CC}$, Wu D, Wang JH, Yang WC, Tseng CH. Association of $\mathrm{C}$-reactive protein and hyperuricemia with diabetic nephropathy in Chinese type 2 diabetic patients. Acta Diabetol. 2009;45(2):127-134. doi:10.1007/s00592-008-0069-0. 